ARTICLE

\title{
Ketamine administration during a critical period after forced ethanol abstinence inhibits the development of time-dependent affective disturbances
}

\author{
Oliver Vranjkovic ${ }^{1,2,3}$, Garrett Winkler ${ }^{3}$ and Danny G. Winder ${ }^{1,2,3,4,5}$
}

Forced abstinence from chronic two bottle-choice ethanol drinking produces the development of negative affective states in female $\mathrm{C} 57 \mathrm{BL} / 6 \mathrm{~J}$ mice. We previously reported that this disrupted behavior is acutely reversed by administration of ketamine 30 min-prior to testing. Here we assessed whether ketamine can be used as an inoculant against the development of abstinencedependent affective disturbances. In parallel, we examined the impact of ketamine administration on long-term potentiation (LTP) in the bed nucleus of the stria terminalis (BNST), a region implicated in affective disturbances. We administered ketamine ( $3 \mathrm{mg} / \mathrm{kg}$ i.p.) to female $\mathrm{C} 57 \mathrm{BL} / 6 \mathrm{~J}$ mice with a history of chronic ethanol drinking at either the onset, two, or 6 days- post-abstinence and observed its impact on affective behavior in the elevated plus maze (EPM), the Novelty Suppressed Feeding Test (NSFT), and the Forced Swim Test (FST). In addition, we assessed BNST synaptic plasticity with field potential electrophysiology two to 3 weeks into abstinence. We found that early abstinence was associated with disrupted behavior on the EPM. Ketamine administered at the onset of forced abstinence prevented both the deficit in early EPM behavior, and the delayed deficits in NSFT and FST. However, ketamine administered either two or 6 days post-abstinence failed to prevent the abstinence-induced affective disturbances. To begin to explore potential alterations in neural circuit activity that accompanies these actions of ketamine, we assessed the impact of ketamine administration at the onset of forced abstinence and measured LTP induction in the BNST. We find that early ketamine administration persistently increased the capacity for LTP within the BNST. These findings suggest a critical period at the onset of forced abstinence in which ketamine inoculation can prevent the development of affective disturbances, in part by enhancing plasticity within the BNST.

Neuropsychopharmacology (2018) 43:1915-1923; https://doi.org/10.1038/s41386-018-0102-0

\section{INTRODUCTION}

Affective disturbances such as generalized anxiety and major depression are associated with alcohol use disorders (AUDs) in humans [1]. Rodent and human studies have shown that negative affective disturbances can develop during withdrawal from chronic contingent alcohol drinking [2-6] and contribute to negative reinforcement-based alcohol intake [6-8]. Current treatment strategies for managing depression largely include blocking either reuptake or breakdown of monoamine neurotransmitters. These therapeutics have drawbacks in that it takes weeks to months for a therapeutic response, and nearly $50 \%$ of patients show full remission [1, 9]. Moreover, drinking alcohol can potentiate the side effects of antidepressants and worsen the symptoms of depression; for example, reuptake inhibitors can increase alcohol seeking behavior [10, 11]. In addition, studies have shown that alcohol can lower the seizure threshold for antidepressants that act as monoamine reuptake inhibitors [12]. Therefore, these limitations make it difficult to treat and manage alcohol withdrawal dependent affective disturbances $[13,10]$.

Recent molecular and behavioral studies have shown that subanesthetic doses of ketamine can elicit rapid, long-lasting antidepressant actions $[9,14,15]$. We recently reported that 2 week withdrawal from 6-weeks of continuous access 2 bottle choice (2BC) ethanol drinking induces disruption in affective disorders as assessed by the novelty suppressed feeding test (NSFT) and forced swim test (FST) that can be blocked by an acute injection of ketamine (3 mg/kg i.p.) 30-min prior to testing [3]. Here we assess the time-dependence of ketamine administration on affective behavior during ethanol forced abstinence. We find that ketamine prevents the development of affective disturbances when administered at the onset of forced abstinence, and not shortly thereafter (2-6 days).

Studies suggest that the GluN2B subunit of the N- methyl- Daspartate (NMDA) receptor participates in regulating affect and in the antidepressant actions of ketamine $[9,14,16]$. Chronic ethanol administration and early withdrawal increase expression of GluN2B in several brain areas, particularly within the central nucleus of the amygdala and bed nucleus of the stria terminalis (BNST) [17], both of which are heavily involved in regulating affect [18-21]. Previously, we found that knockdown of GluN2B-within the BNST produces antidepressant-like actions similar to ketamine [22] and that GluN2B is necessary for long-term potentiation (LTP)

\footnotetext{
${ }^{1}$ Department of Molecular Physiology and Biophysics, Vanderbilt University School of Medicine, Nashville, TN, USA; ${ }^{2}$ Vanderbilt Center for Addiction Research, Vanderbilt University School of Medicine, Nashville, TN, USA; ${ }^{3}$ Vanderbilt Brain Institute, Vanderbilt University School of Medicine, Nashville, TN, USA; ${ }^{4}$ Department of Psychiatry and Behavioral Sciences, Vanderbilt University School of Medicine, Nashville, TN, USA and ${ }^{5}$ Department of Pharmacology, Vanderbilt University School of Medicine, Nashville, TN, USA Correspondence: Danny G. Winder (Danny.winder@vanderbilt.edu)
}

Received: 28 November 2017 Revised: 16 April 2018 Accepted: 16 May 2018

Published online: 24 May 2018 
within the BNST [23]. Furthermore, we have previously shown that non-contingent chronic intermittent ethanol enhances LTP within the BNST which is dependent on the GluN2B subunit [23]. However, no studies have looked at LTP within the BNST during withdrawal after contingent 2-bottle choice ethanol drinking. Here we show that withdrawal from $2 B C$ ethanol drinking decreases the early component of LTP within the BNST. Further, administration of ketamine at the onset of forced abstinence, but not shortly thereafter (2-6 days) facilitated later LTP induction.

\section{MATERIALS AND METHODS}

Animals

One-hundred and eighty-two female C57BL/6J mice were used for this study (The Jackson Laboratory; Bar Harbor, ME). Mice were delivered at 7 weeks of age, group housed (5 mice/cage), and allowed to acclimate to the colony for 1 week. After the acclimation period, mice were singly housed. Mice were maintained on a $12 \mathrm{~h}$ light/dark cycle (lights on at $0700 \mathrm{~h}$ ) under controlled temperature $\left(20-25^{\circ} \mathrm{C}\right.$ ) and humidity (30-50\%) levels. Treatments were approved by the Vanderbilt Animal Care and Use Committee.

\section{Drugs}

Ketamine HCL (Patterson Veterinary, Devens, MA) was diluted in $0.9 \%$ saline and administrated at $3 \mathrm{mg} / \mathrm{kg}$ i.p.

Two-bottle choice (2BC) ethanol drinking Procedures were conducted as previously described [3]. Briefly, mice underwent an $\mathrm{EtOH}$ ramp procedure in which mice received either tap water, or ethanol in $30 \mathrm{~mL}$-conical tubes. Ethanol was administered in increasing concentration 3\% for 3 days; $7 \%$ for 6 days, then $10 \%$. Mice were kept on $10 \%$ for four-weeks and then moved into the forced abstinence period in which both conical tubes contained tap water. Mice were kept on water for the remainder of the experiment. Ethanol and water sipper positions were switched weekly to avoid position bias. Conical tubes and mice were weighed every 48-h. A drip cage, with one ethanol conical tube and one water conical tube was used to control for natural drip. Preference percentage was measured as ((EtOH solution consumed (g) - Drip)/(total consumed (g)-Drip)) $\times 100$.

Timeline for ketamine administration during abstinence in order to prevent the development for affective disorders

Following 2BC ethanol drinking mice were moved to forced abstinence. We administered ketamine at three different time points during forced abstinence. Ketamine $3 \mathrm{mg} / \mathrm{kg}$ i.p. was administered either at the onset of abstinence or as soon as the bottles were switched from ethanol to water (day 42), 2-days after the onset of abstinence (day 44), or 6-days (day 48) after the onset of abstinence. Ketamine was administered one to three hours after the onset of the light cycle in all three conditions. Mice were tested for affective disturbances during the abstinence period. Mice were tested on the elevated plus maze (EPM) 24-h after ketamine administration. Novelty-Suppressed Feeding (NSFT) and FST were conducted 2 weeks and 3 weeks after the onset of abstinence respectively (day 56-63).

Elevated plus maze (EPM)

The maze is elevated $55 \mathrm{~cm}$ above the ground and consists of two open arms and two closed arms $(30.5 \times 6.5 \mathrm{~cm} ; 16 \mathrm{~cm}$ closed arm height) with a $5 \times 5 \mathrm{~cm}$ open center zone. The center, and each arm was illuminated to 50 lux. Mice were brought into the testing room and allowed to acclimate for one hour. Mice were placed in the center facing alternate arms and recorded for $5 \mathrm{~min}$. Mice were visualized, recorded, and tracked by camera using AnyMaze software as previously described [3] (Stoelting Co, Wood Dale, IL).
Forced Swim Test (FST)

Mice were brought into the testing room and allowed to acclimate for one hour. Mice were then exposed to a 6-min forced swim in a transparent cylinder filled with water $\left(23-25^{\circ} \mathrm{C}\right)$. Swim sessions were recorded and a blind observer scored the last 4-min for immobility as previously described [3]. All of the mice in this group had undergone both EPM and NSFT prior to the FST.

Novelty-Suppressed Feeding Test (NSFT)

NSFT was conducted as previously described [3]. Forty-eight-hours prior to NSFT, mice were food deprived with a free-feeding window between hours 23-25. In order to acclimate the mice to testing conditions, mice were brought into the testing room 1-h prior to testing. In the center of the room, directly below the video camera, a white box $(32 \times 32 \times 32 \mathrm{~cm})$ was covered with $2 \mathrm{~cm}$ of fresh bedding material. A standard normal mouse chow (LabDiet5L0D; West Durham, NC) pellet was placed in the center. Mice were placed into one corner of the box and allowed to explore the box for up to $20 \mathrm{~min}$. Mice were visualized, recorded, and tracked by Any Maze software as previously described. Latency was defined by the duration (in seconds) it took for a mouse to take the first bite of the chow. The video was stopped, and the mouse was immediately removed from the box and returned to the home cage. All of the mice in this group had previously undergone a single EPM exposure $24 \mathrm{~h}$ after saline or ketamine administration.

Slice preparation

Mice were transported from the colony room to the laboratory and kept in a sound-attenuated cubicle (Med Associates; (St. Albans City, VT) for one hour before slicing. Mice were anesthetized with isoflurane and then transcardially perfused with ice cold sucrose artificial cerebrospinal fluid solution (aCSF) containing (in mM): 194 sucrose, $20 \mathrm{NaCl}, 4.4 \mathrm{KCl}, 2 \mathrm{CaCl}_{2}, 1 \mathrm{MgCl}_{2}$, $1.2 \mathrm{NaH}_{2} \mathrm{PO}_{4}, 10.0$ glucose, and $26.0 \mathrm{NaHCO}_{3}$ saturated with $95 \%$ $\mathrm{O}_{2} / 5 \% \mathrm{CO}_{2}$. Brains were quickly removed and placed in ice-cold sucrose. Three hundred micometer thick coronal slices were prepared using a Leica Tissue Slicer (Leica Biosystems Inc.; Buffalo Grove, IL). After dissection, slices were transferred to a holding chamber containing headed $\left(29^{\circ} \mathrm{C}\right.$ ) and oxygenated $95 \% \mathrm{O}_{2} / 5 \%$ $\mathrm{CO}_{2}$ aCSF containing (in mM) $124 \mathrm{NaCl}, 4.4 \mathrm{KCl}, 2 \mathrm{CaCl}_{2}, 1.2 \mathrm{MgSO}_{4}$, $1 \mathrm{NaH}_{2} \mathrm{PO}_{4}, 10.0$ glucose, and $26 \mathrm{mM} \mathrm{NaHCO}$ for at least onehour.

Field potential recordings

Slices where allowed to equilibrate for at least $1 \mathrm{~h}$. Slices were then moved to an interface-recording chamber, where they were perfused with heated $\left(29^{\circ} \mathrm{C}\right)$ and oxygenated aCSF at a rate of 2 $\mathrm{mL} / \mathrm{min}$. A glass recording electrode filed with ACSF and a bipolar nichrome stimulating electrode were placed in the dIBNST to elicit an extracellular field response. Baseline responses to a stimulus (50us) at an intensity that produced $40 \%$ of the maximum response were recorded for $20 \mathrm{~min}$ at a rate of $0.05 \mathrm{~Hz}$. After the 20 min baseline period, LTP was induced with two trains of $100 \mathrm{~Hz}$, $1 \mathrm{~s}$ tetanus delivered with a $20 \mathrm{~s}$-intertrain interval at the same intensity as base line test pulse. The N1 was monitored throughout the experiment, and if the $\mathrm{N} 1$ changed by more than $20 \%$ the slice was discarded. Analyses were made from the percent change of the $\mathrm{N} 2$ from baseline 0-10 min after tetanus and $51-60 \mathrm{~min}$ after tetanus.

\section{Statistics}

All data are represented as mean \pm SEM. All statistics were run using Prism 6 (Graphpad, La Jolla, CA). Differences between groups were assessed using $t$-tests and one-way ANOVA with significance set at $p=0.05$. When significant main effects were obtained using ANOVA tests, a Dunnett's post hoc comparisons between groups was performed. 


\section{RESULTS}

Six-week chronic 2 bottle choice $(2 \mathrm{BC})$ alcohol drinking in female C57BL/6J mice used for the various experiments are shown in Table 1. All groups of mice showed an increase in preference and intake as previously shown [3]. Ethanol drinking remained constant during the last four-weeks when the mice had access to $10 \% \mathrm{EtOH}$.

Female mice developed affective disturbances in abstinence from 6-week 2BC ethanol drinking

The effect of forced abstinence on affective disturbances was evaluated in female $\mathrm{C} 57 \mathrm{BL} / 6 \mathrm{~J}$ mice following chronic 6-week alcohol drinking (Fig. 1a). In previous studies, we and others have shown that prolonged forced abstinence (i.e., 2 weeks or later) induces significant differences in depressive-like behaviors, such as FST, sucrose preference test, and NSFT, but not in tests more sensitive to anxiety such as the EPM and light-dark box [3-5]. In these previous studies, however, anxiety-like behavior was not assessed during early forced abstinence ([3]; Pang et al. 2016; Pang et al. 2016; [2]). Thus, in the present study, 22 water-drinking mice, and 35 ethanol-drinking mice were tested on the EPM 24-h

\begin{tabular}{|c|c|c|c|c|}
\hline & \multicolumn{2}{|c|}{ Preference $(\% \mathrm{EtOH})$} & \multicolumn{2}{|c|}{ Consumption (g/kg EtOH) } \\
\hline & Mean & SEM & Mean & SEM \\
\hline Week 1 & 62.26 & 5.34 & 15.223 & 5.320 \\
\hline Week 2 & 62.70 & 7.88 & 14.553 & 6.540 \\
\hline Week 3 & 75.00 & 3.98 & 17.430 & 4.550 \\
\hline Week 4 & 80.43 & 4.56 & 21.444 & 2.320 \\
\hline Week 5 & 80.18 & 3.23 & 21.55 & 3.450 \\
\hline Week 6 & 84.32 & 2.22 & 23.356 & 4.320 \\
\hline
\end{tabular}

after the onset of forced abstinence (day 43). In this study, mice with a history of ethanol drinking spent significantly less time on the open arms (Fig. 1b; Student's $t$-test, $t(55)=3.719, p<0.0001$ ). Student's $t$-test revealed no significant effect of drinking on distance traveled on the EPM (data not shown; Student's $t$-test, $t(55)=0.8194, p<0.42$ ) indicating that locomotor-activity was not impaired in this group of mice. Thirteen water-drinking mice, and 18 ethanol-drinking mice were tested for a depressive phenotype two-weeks (day 56) after the onset of forced abstinence using NSFT, all of these mice were exposed to the EPM two-weeks prior. This experiment revealed that mice with a history of ethanol drinking took significantly longer to feed (Fig. 1c; Student's $t$-test, $t(29)=5.539, p<0.0001)$

Ketamine administration at the onset of abstinence prevented ethanol abstinence induced affective disturbances

Previously, we have shown that an acute injection of ketamine is able to reverse affective disturbances induced by prolonged abstinence from $2 \mathrm{BC}$ ethanol drinking when administered 30-min prior to testing [3]. Our goal in the present study was to identify a time-course window in which ketamine administration is effective in preventing the development of affective disturbances. First, we tested whether ketamine given at the onset of abstinence (at the same time the ethanol was removed and changed to water: day 42) could prevent the development of affective disturbances. Nineteen mice underwent 2BC water drinking, and 25 mice underwent 2BC ethanol drinking for 6 weeks. Thirty-mice (19 water drinkers and 11 ethanol drinkers) received a saline injection at the onset of abstinence, and 14 ethanol-drinking mice received an injection of ketamine ( $3.0 \mathrm{mg} / \mathrm{kg}$ i.p.) at the onset of abstinence (day 42). Mice were subsequently tested for the development of affective disturbances (Fig. 2a).

Twenty-four hours after the onset of abstinence (day 43), mice were tested on the EPM. One-way ANOVA revealed a significant effect of drinking on time spent in the open $\operatorname{arm}(F(2,41)=6.443$; $p=0.0037)$. Dunnett's post hoc revealed that saline-injected ethanol-drinking mice spent less time in the open arm compared

A.

\begin{tabular}{|c|c|c|c|}
\hline $3 \%$ EtOH & 7\% EtOH & $10 \%$ EtOH & Forced Abstinence (Water) \\
\hline
\end{tabular}
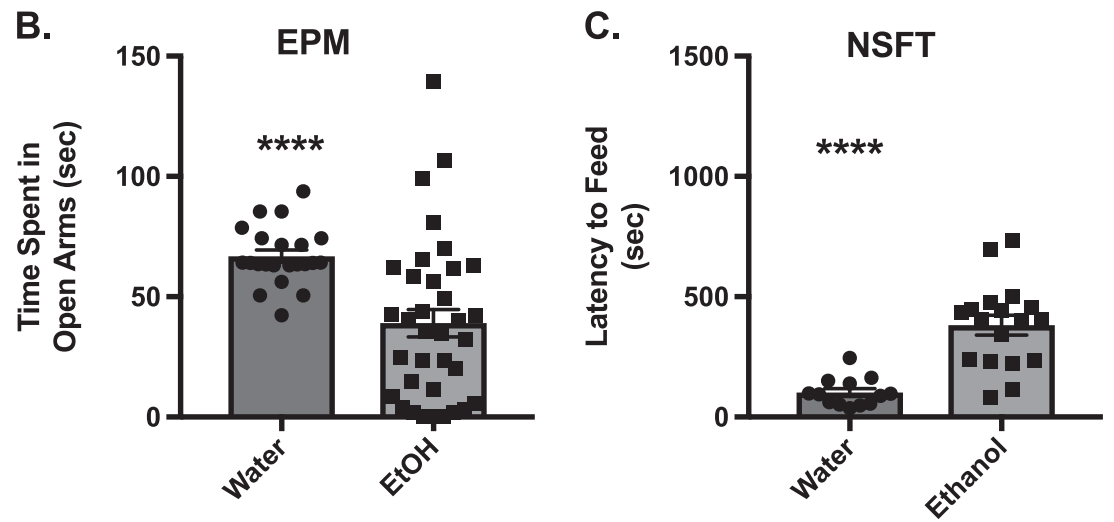

Fig. 1 Female mice with a history of chronic six-week long ethanol drinking develop affective disturbances. Mice were tested on the EPM twenty-four hours after the onset of abstinence, and NSFT two weeks after the onset of abstinence (a). Mice with a history of ethanol drinking displayed less time spent on the open arms compared to mice with a history of water drinking (b). Mice with a history of ethanol-drinking displayed a longer latency to feed in NSFT compared to mice with history of water-drinking (c). Time and distance are shown as the mean \pm SEM. ${ }^{* * * *} p<0.0001$ compared to saline-ethanol drinkers; analysis of variance ( $t$-test) 
A.

Day 1 . .9. .42. 56
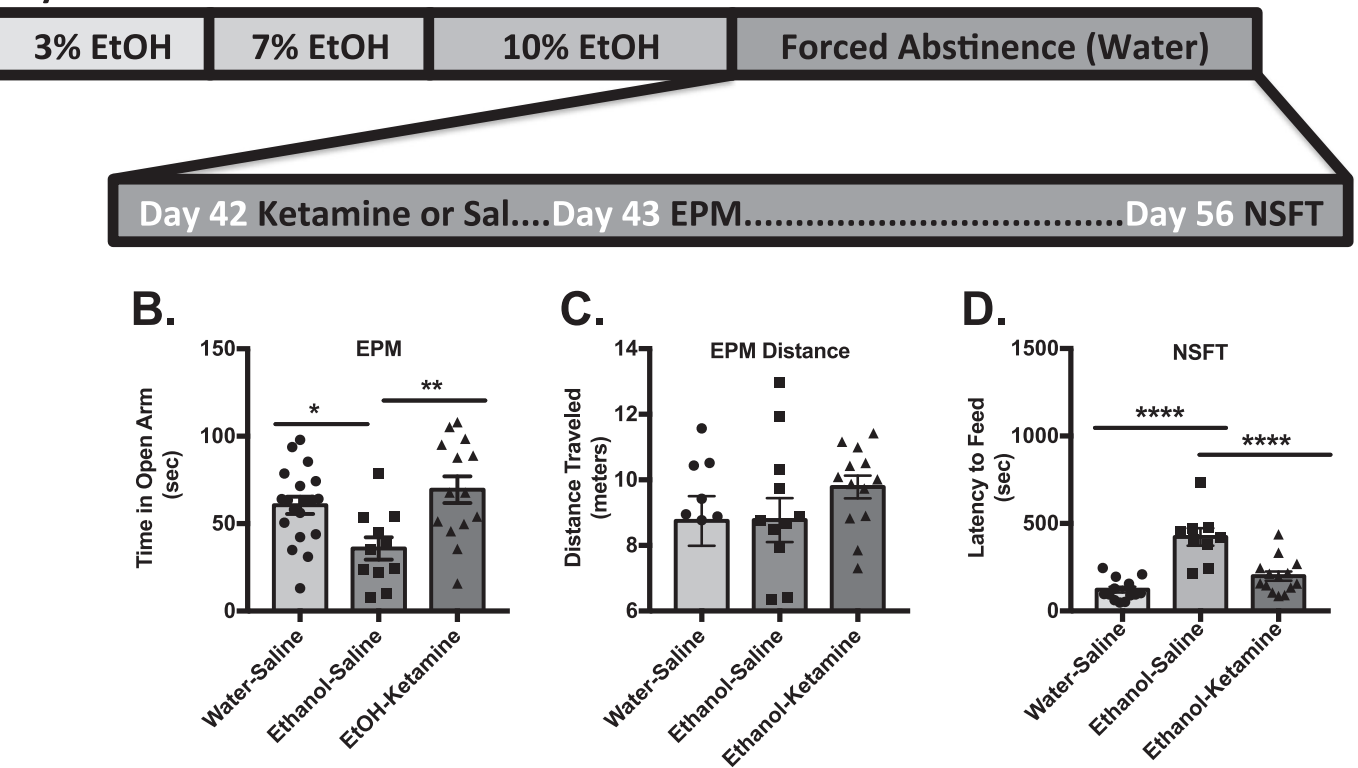

Fig. 2 A single low dosage of ketamine $(3.0 \mathrm{mg} / \mathrm{kg}$ i.p.) at the onset of abstinence after six-weeks of chronic drinking prevents the development of affective disturbances. Representative figure for $2 \mathrm{BC}$ ethanol drinking followed by chronic abstinence, ketamine was administered at the onset of abstinence (a). Ketamine-injected mice showed a significant increase in time spent on open arms compared to saline injected ethanol drinking mice (b). Distance traveled on the EPM did not differ amongst the three groups (c). Mice develop affective disturbances two-weeks after abstinence as measured by NSFT. This was reversed by an administration of ketamine at the onset of abstinence (d). Time and distance are shown as the mean \pm SEM ( $n=6-12$ /group). ${ }^{*} p<0.05,{ }^{* *} p<0.01,{ }^{* * * *} p<0.0001$ compared to saline-ethanol drinkers; analysis of variance (one-way ANOVA with Dunnett's post hoc test)

to saline-injected water-drinking mice $(p=0.0172)$, and ketamineinjected ethanol-drinking mice $(p=0.0172$ ) (Fig. 2b). One-way ANOVA revealed no significant effect on drinking, or treatment on distance traveled on the $\operatorname{EPM}(F(2,41)=1.086 \quad p=0.9993)$ indicating that locomotor-activity was not impaired in this group of mice (Fig. 2c).

Next, mice underwent the NSFT 2 weeks following the onset of abstinence (day 56). One-way ANOVA revealed a significant effect of drinking $(F(2,34)=24.48 ; p<0.0001)$ in latency to feed. Dunnett's post hoc test revealed that saline-injected ethanol drinkers had a longer latency to feed compared to saline-injected water drinkers $(p=0.0001)$, and a longer latency to feed compared to ketamine-injected ethanol-drinkers $(p=0.0001)$. Saline-injected water drinkers, and ketamine-injected ethanol drinkers did not show a significant difference ( $p=0.42)$ (Fig. $2 d$ ).

Ketamine administration two-days after the onset of abstinence failed to prevent ethanol abstinence induced affective disturbances Next, we sought to determine whether ketamine administered two-days (day 44) into abstinence could also prevent the development of affective disturbances. Ten-mice underwent water $2 \mathrm{BC}$ and 20 mice underwent ethanol $2 \mathrm{BC}$ for six-weeks. Two-days into forced abstinence, 11 mice (five water drinkers and six ethanol drinkers) received an i.p. injection of saline, and six ethanol-drinking mice received an i.p. injection of ketamine (3.0 $\mathrm{mg} / \mathrm{kg}$ ). Mice were subsequently tested for the development of affective disturbances (Fig. 3a).

Twenty-four hours after the saline or ketamine injection ( 3 days after the onset of abstinence; day 45) mice were tested on the EPM. One-way ANOVA revealed a significant effect of drinking on time spent in the open $\operatorname{arm}(F(2,17)=4.904 ; p=0.0208)$. Dunnett's post hoc revealed that saline-injected ethanol-drinking mice spend less time in the open arm compared to saline-injected water-drinking mice ( $p=0.0174)$ but had no significant difference in open time with ketamine-injected ethanol-drinking mice ( $p=$
0.78) (Fig. 3b). One-way ANOVA revealed no-significant effect on water drinking, or treatment on distance traveled on the EPM ( $p=$ 0.9960 ) indicating that locomotor-activity was not impaired in this group of mice (Fig. 3c).

Next, a total of 23 mice (six water drinkers and 17 ethanol drinkers) underwent NSFT two-weeks after the onset of abstinence (day 56). One-way ANOVA revealed no significant effect of drinking in latency to feed (Fig. $3 d ; F(2,19)=1.337 ; p, 0.28$ ).

Ketamine administration six-days after the onset of abstinence failed to prevent ethanol abstinence induced affective disturbances

To further develop a timeline, ketamine or saline was administered six-days into abstinence (day 48) to determine if it is effective in preventing the development of affective disturbances. Nine mice underwent water $2 \mathrm{BC}$ and 30 mice underwent ethanol 2BC. Sixdays into forced abstinence, 22 ( 9 water drinkers and 13 ethanol drinkers) received an i.p. injection of saline, and 17 mice received an i.p. injection of ketamine $(3.0 \mathrm{mg} / \mathrm{kg})$. Mice were subsequently tested for the development of affective disturbances (Fig. 4a).

Twenty-four hours after the saline or ketamine injection (day 49) mice were tested on the EPM. One-way ANOVA revealed a significant effect of drinking on time spent in the open arm ( $F$ $(2,36)=4.42 ; p=0.0192)$. Dunnett's post hoc revealed that salineinjected ethanol-drinking mice spent less time in the open arm compared to saline-injected water-drinking mice $(p=0.0116)$ but no difference was observed in time spent in the open arms with the ketamine-injected ethanol-drinking mice ( $p=0.587)$ (Fig. 4b). One-way ANOVA revealed no significant effect on drinking, or treatment on distance traveled on the EPM $(p=0.7114)$ indicating that locomotor-activity was not impaired in this group of mice (Fig. 4c).

Next, mice were tested 2 weeks following the onset of abstinence using the NSFT (day 56). One-way ANOVA revealed a significant effect of drinking $(F(2,41)=11.67 ; p<0.0001)$ in latency to feed. Dunnett's post hoc test revealed that saline-injected 
A.

Day 1.

3.

..9.

. .42 .

.56

\begin{tabular}{|l|l|l|l}
\hline $3 \% \mathrm{EtOH}$ & $7 \% \mathrm{EtOH}$ & $10 \% \mathrm{EtOH}$ & Forced Abstinence (Water)
\end{tabular}

Day 44 Ketamine or Sal....Day 45 EPM............................. Day 56 NSFT
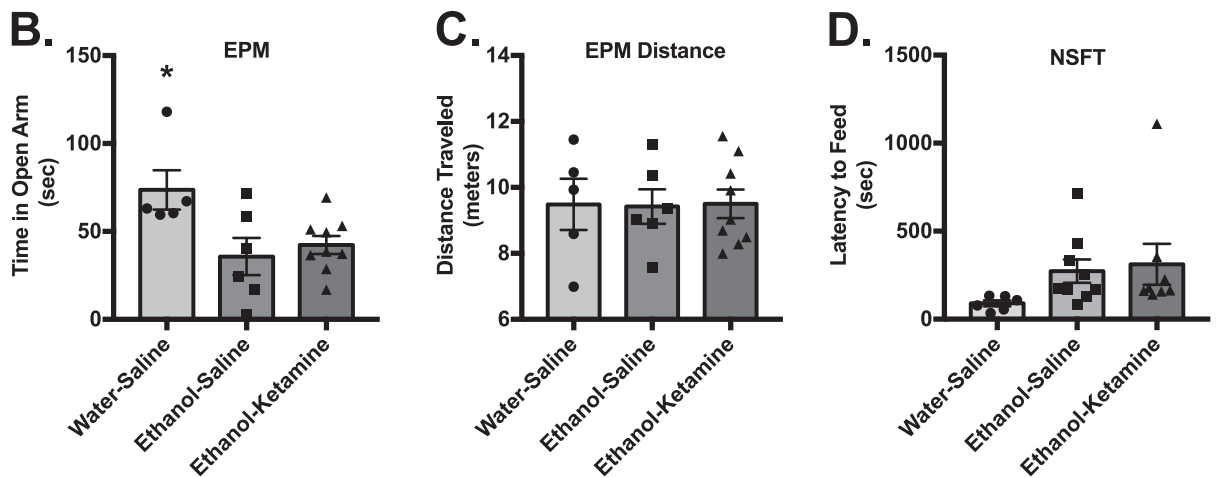

Fig. 3 A single low dosage of ketamine $(3.0 \mathrm{mg} / \mathrm{kg}$ i.p.) 2 days into abstinence after six-weeks of chronic drinking failed to prevent the development of affective disturbances. Representative figure for $2 \mathrm{BC}$ ethanol drinking followed by chronic abstinence. Ketamine was administered 2 days after the onset of abstinence (a). Ketamine failed to prevent abstinence induced anxiety (b). Distance traveled on the EPM did not differ amongst the three groups (c). Ketamine failed to block abstinence induced affective disturbances as measured by NSFT (d). Time and distance are shown as the mean \pm SEM ( $n=6-12 /$ group). ${ }^{*} p<0.05,{ }^{* *} p<0.01,{ }^{* * * *} p<0.0001$ compared to saline-ethanol drinkers; analysis of variance (one-way ANOVA with Dunnett's post hoc test)

A.

Day 1

3......

9..

.56
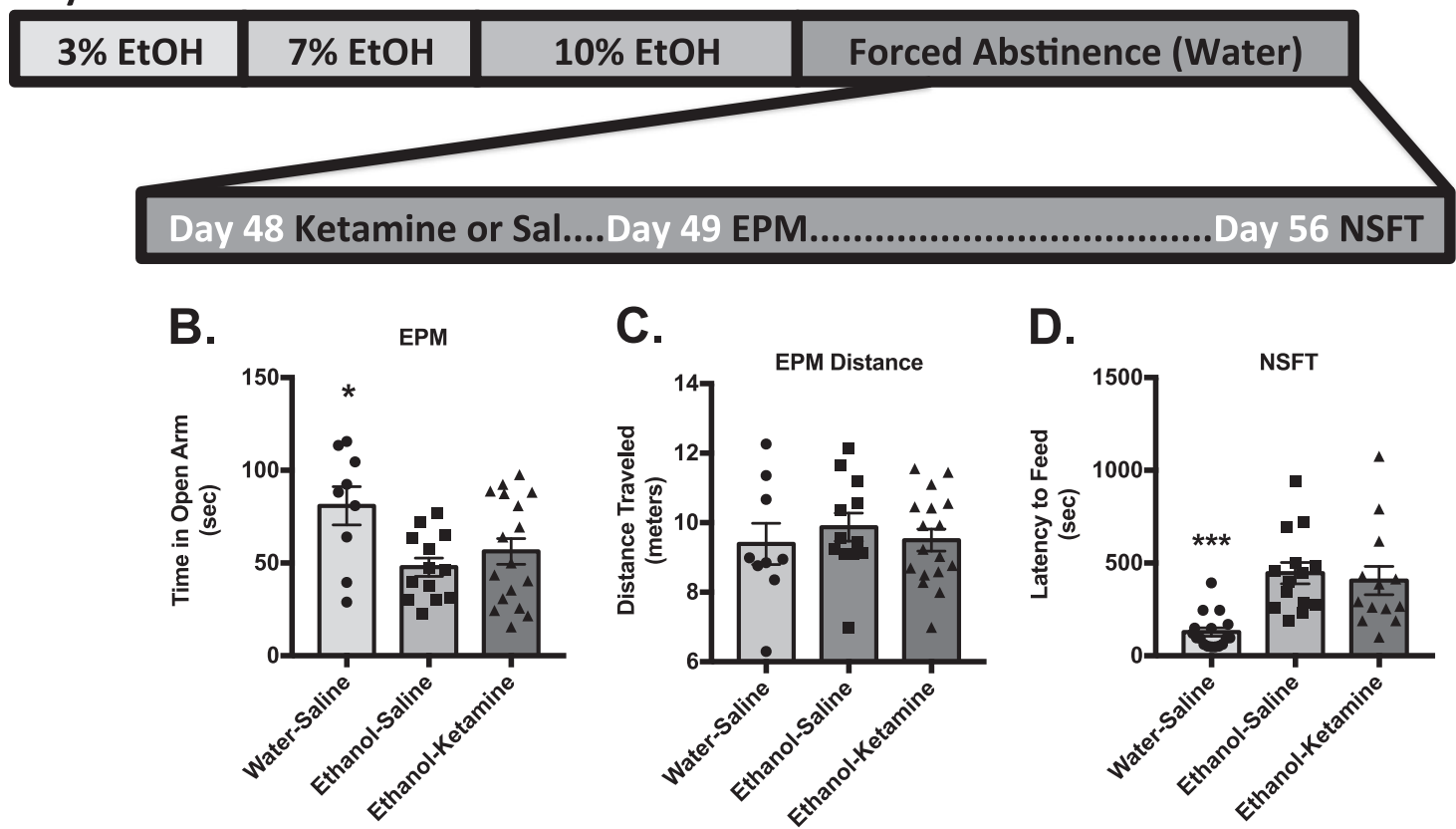

Fig. 4 A single low dosage of ketamine $(3.0 \mathrm{mg} / \mathrm{kg}$ i.p.) 6 days into abstinence after six-weeks of chronic drinking failed to prevent the development of affective disturbances. Representative figure for $2 \mathrm{BC}$ ethanol drinking followed by chronic abstinence. Ketamine was administered 6 days after the onset of abstinence (a). Ketamine failed to prevent abstinence induced anxiety (b). Distance traveled on the EPM did not differ amongst the three groups (c). Ketamine failed to block abstinence induced affective disturbances as measured by NSFT (d). Time and distance are shown as the mean \pm SEM ( $n=6-12$ /group). ${ }^{*} p<0.05,{ }^{* *} p<0.01,{ }^{* * *} p<0.0001$ compared to saline injected-ethanol drinkers; analysis of variance (one-way ANOVA with Dunnett's post hoc test) 

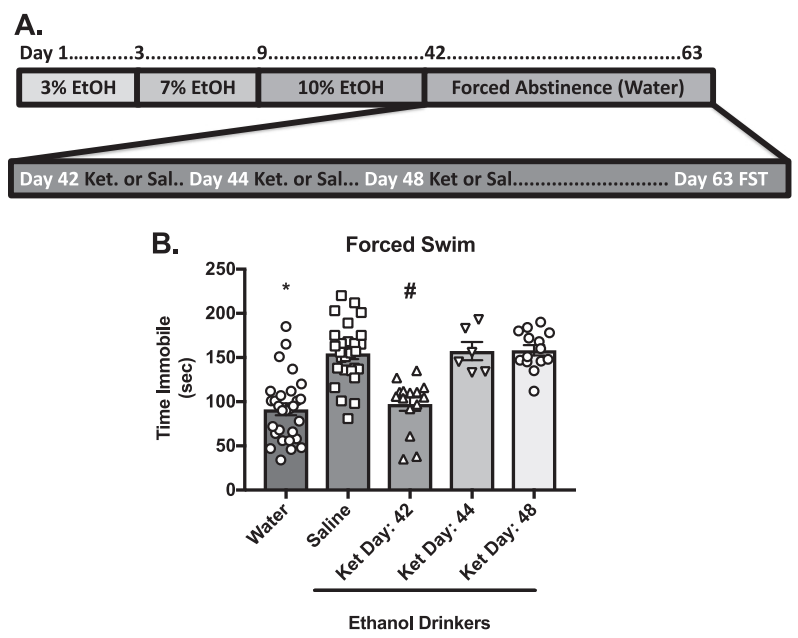

Fig. 5 Ketamine prevented affective disturbances in the FST only when administered at the onset of abstinence. Representative figure for two-bottle choice ethanol drinking followed by chronic abstinence. Mice received an injection of ketamine at three different time followed by a FST three weeks after the onset of abstinence (a). Ketamine administration at the beginning of abstinence prevented affective disturbances as assessed by the FST (b). Immobility time is shown as the mean \pm SEM ( $n=6-29 /$ group). ${ }^{*} p<0.0001$ and $\# p<$ 0.0001 compared to saline injected-ethanol drinkers; analysis of variance (one-way ANOVA with Dunnett's post hoc test)

ethanol drinkers had a longer latency to feed compared to salineinjected water drinkers ( $p=0.0002)$, but not a significant change in latency to feed compared to ketamine-injected ethanol-drinkers $(p=0.82)$ (Fig. 4d).

Ketamine administration at the onset of abstinence prevents ethanol abstinence induced disturbances as assessed by the FST The FST, a pharmacologically established proxy for depression-like behavior, was used as an additional test for measuring affective disorders. We repeated earlier findings showing that FST immobility in EtOH mice following 18 days of forced abstinence increases FST immobility time [3]. One-week after the NSFT test, mice underwent the FST test. One-way ANOVA shows that ethanol drinking increases immobility time (Fig. 4b. ${ }^{*} p<0.0001$ ). Here we assessed the effectiveness of ketamine administration during the three-time points used above on FST performed in later abstinence (Fig. 4a). Ketamine prevented the development of FST disturbances only when it was administered at the beginning of forced abstinence (day 42; Dunnett's post hoc $\# p<0.0001$ ) (Figure 5).

Ketamine administration at the onset of abstinence persistently increases the capacity for plasticity within the BNST

The BNST is a region heavily involved in affective behavior that also is thought to play an important role in alcohol withdrawalrelated phenotypes $[24,25]$. In parallel with the time-dependent ketamine injection studies on affective behavior, we also assessed the time dependency of ketamine administration on the capacity for LTP induction within the BNST. We thus assessed the effect of early abstinence ketamine (day 42), and ketamine administered six-days (day 48) into abstinence on BNST LTP induction two to 3 weeks after the onset of abstinence. Fifteen mice underwent 2BC ethanol drinking followed by forced abstinence, and four mice underwent $2 \mathrm{BC}$ water drinking for 8 weeks. At the onset of abstinence, five ethanol drinking mice received an injection of saline, and another five received an injection of ketamine $(3.0 \mathrm{mg} /$ $\mathrm{kg}$ ). Six days into abstinence (day 48), another five ethanol drinking mice received an injection of ketamine. As a control, we performed LTP recordings in mice with a history of $2 \mathrm{BC}$ water drinking. Four mice underwent $2 \mathrm{BC}$ water drinking for six-weeks and received an injection of saline on the 42nd day of drinking. We recorded LTP within the BNST in all mice two to three weeks into abstinence (day 56-63) (Fig. 6a).

Tetanization produced LTP in the BNST of mice with a history of water $2 B C$, consistent with previous studies $[23,26]$. LTP was also induced in slices obtained from ethanol abstinent mice. A marked decrease in an early component of LTP (referred to here as Shortterm Potentiation, STP) was observed in these experiments, along with a very modest but statistically significant reduction in the later component. We compared the first ten minutes after tetanization and observed that animals with a history of ethanol drinking showed significantly lower STP compared to animals with a history of ethanol 2BC (Fig. 6c: Student's t-test $t(18)=5.732$, $p<0.0001)$. When we compared the last ten minutes of the recording, we found a very modest but significant decrease in LTP in slices from the ethanol abstinent mice. (Fig. 6d; Student's $t$-test $t$ $(18)=3.57, p<0.0022$ ).

We next examined the impact of ketamine administration at either the onset of abstinence (day 42), or 6 days (day 48) into abstinence on measured BNST LTP two to three weeks after abstinence (day 56-63). We found that mice with a history of ethanol drinking that received an injection of ketamine at the onset of abstinence (day 42) had a robust increase in LTP compared to ethanol drinking mice that received an injection of ketamine six-days into abstinence (day 48) (Fig. 6e). We again compared the first ten minutes after tetanization and found that ethanol drinking mice that got an injection of ketamine 6 days into abstinence had significant lower STP compared to ethanol drinking mice that got an injection of ketamine at the onset of abstinence (Fig. 6f; Student's $t$-test $t(18)=10.56, p<0.0001$ ). When we compared the last ten minutes of the recording, we found that ethanol drinking mice that got an injection of ketamine at the onset of abstinence-maintained potentiation to a higher degree compared to animals that got ketamine on the 6th of abstinence (Fig. 6g; Student's $t$-test $t(18)=15.02, p<0.0001)$. We were also interested to see if potentiation differed in $2 \mathrm{BC}$ ethanol drinking animals that got an injection of saline at the onset of abstinence differed from the ethanol drinking animals that got an injection of ketamine on the $6^{\text {th }}$ day of abstinence. Student's t-test showed no significant difference 10-min after the onset of tetanization (Student's $t$-test $t(18)=1.351, p=0.58$ nor a significant difference in maintain potentiation (Student's $t$-test $t(18)=0.1933, \mathrm{p}=0.19$ ) (data not shown).

\section{DISCUSSION}

Our results provide a key extension of earlier findings that ketamine can block affective disturbances brought about by forced abstinence following six-weeks of $2 \mathrm{BC}$ ethanol drinking. In particular, we found that ketamine prevents the development of affective disturbances if it is administered at the onset, but not 2- or 6-days, after the beginning of abstinence. This is particularly striking since the 2 and 6-day post injections are closer in time to the affective testing than the effective dose at the onset of forced abstinence. Concurrent with these findings, we observed that forced abstinence from six-week 2BC choice ethanol drinking reduced an early component of LTP referred to as STP. Ketamine administered at the onset of abstinence, but not 6 days later rescued the STP deficit and overall increased the capacity for plasticity within the BNST. Our results suggest, for the first time to our knowledge, that ketamine may need to be administered at a specific time point during abstinence in order to effectively treat and manage alcohol use dependent affective disturbances. These data thus suggest a "critical period" during which ketamine is effective in preventing the development of alcohol abstinence induced affective disturbances. 
A.

\begin{tabular}{|c|c|c|c|}
\hline $3 \%$ EtOH & 7\% EtOH & $10 \%$ EtOH & Forced Abstinence (Water) \\
\hline
\end{tabular}

B.
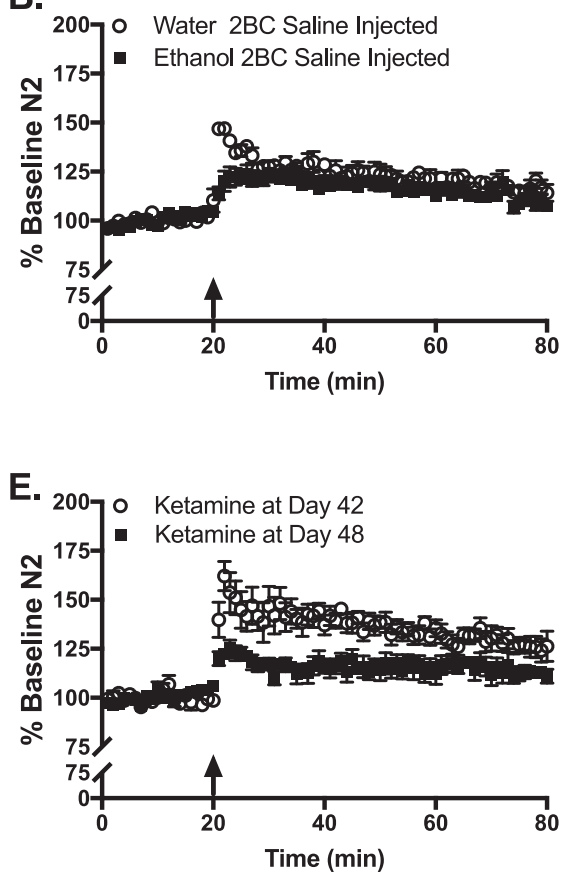

c.

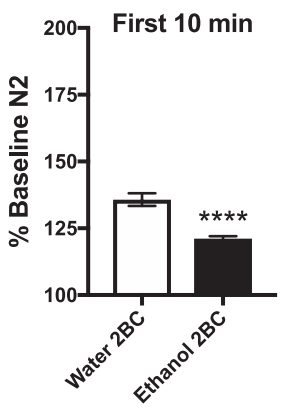

F.

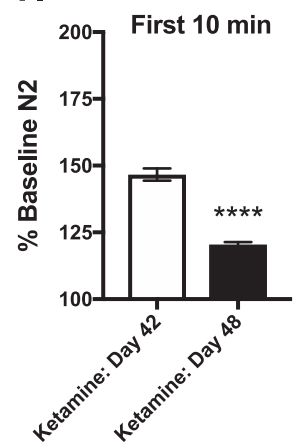

D.

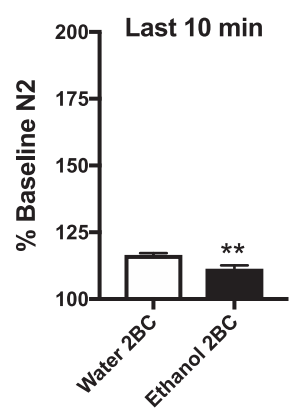

G.

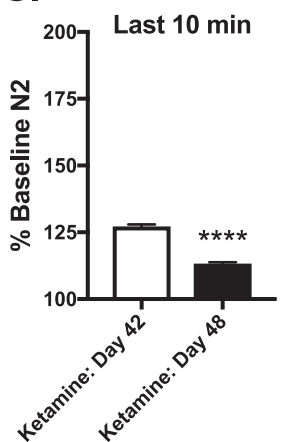

Fig. 6 Ketamine administration at the onset of abstinence persistently increases the capacity for plasticity within the BNST. Representative figure for $2 \mathrm{BC}$ ethanol drinking followed by chronic abstinence and field potential electrophysiology (a). Averaged time course of synaptic field potentials after high-frequency stimulation (arrow depicts two 1-s rains at $100 \mathrm{~Hz}$ ) in the dlBNST from water-drinkers (white circles) or ethanol-drinkers (black squares) (b). Summary bar graph of averaged field potentials 21-30 min (First Ten) post-tetanus (c) and 51-60 min (Last Ten) post-tetanus (from time course in b) (d). Averaged time course of synaptic field potentials after high-frequency stimulation (arrow depicts two 1-s rains at $100 \mathrm{~Hz}$ ) in the dIBNST from ethanol-drinkers injected with ketamine at the onset of abstinence (white circles) or ethanoldrinkers injected with ketamine 6 days into abstinence (black squares) (e). Summary bar graph of averaged field potentials 21-30 min (First Ten) post-tetanus (f) and 51-60 min (Last Ten) post-tetanus (from time course in e) (g). \%Baseline N2 are shown as the mean \pm SEM ( $n=8-13$ / slices between 10 mice) ${ }^{* * *} p<0.0001$ compared to ethanol drinkers with an injection of saline (analysis of variance $t$-test)

The development of affective disturbances

Non-contingent models of rodent alcohol administration have shown that anxiety-like behavior is established during early withdrawal [27], and recent work has shown that prolonged abstinence from contingent ethanol drinking facilitates the development of depression-like behavior $[3,5,6,28]$. Studies have shown that shift from anxiety-like to depressivelike behavior may predominate in females after prolonged abstinence ([3]; Holleran and Winder 2017; [4, 5]) after continuous access contingent ethanol drinking. Here we show that an anxiety-like phenotype on the EPM can be observed 7 days into absence, but this EPM behavior dissipates by fourteen days [3]. While our current study does use NSFT latency measurement as an indicator of depression-like behavior, it is important to note that NSFT can also be used to measure alterations in anxiety-like behavior since both antidepressants and anxiolytic agents may reduce latency to feed $[29,30]$. Therefore, we utilized immobility time in the FST test as an additional measure for depression-like behavior. FST immobility is also increased in prolonged abstinence from following $2 \mathrm{BC}$ ethanol drinking [3-5].
Time-dependent actions of ketamine

"Self-medication" to relieve both anxiety and depressive symptoms is proposed as a driving force for alcohol abuse in some cases, potentially leading to relapse to alcohol seeking during abstinence [8]. Current medications are not effective in treating affective disturbances in a timely fashion. Our results suggest that success in treating AUDs, may involve timedelimited delivery periods for pharmacological agents like ketamine. Previous studies have shown that ketamine rapidly reverses affective disorders $[3,14,22,31]$, however this may not be long lasting since here we show that rodents injected with ketamine at 2-dys or 6-days into abstinence still develop affective disturbances as assessed by NSFT and FST. When we injected ketamine at the onset of abstinence, affective disturbances were not present two- and three-weeks into abstinence. This suggests that the acute, rapid effects of ketamine may temporarily treat affective disturbances; the long-lasting effect of ketamine is dependent on the period of administration. Similar ketamine time-dependent findings have been shown in both stress-induced depressive disorders, and contextual fear conditioning $[32,33]$. 


\section{Ketamine action in abstinence-associated negative affect}

While the specific anti-depressive mechanisms of ketamine and other rapid anti-depressants are being actively investigated (for review see: [34-36]), pre-clinical studies have suggested that antagonism of GluN2B containing NMDARs with Ro-25-6981 decreases depressive-like behavior in the force swim test $[16,37$, 38], NSFT [31, 37, 38], and novelty-induced hypophagia [22]. In addition, another GluN2B-NMDA receptor antagonist, ifenprodil, has been shown to reverse chronic unpredictable stress-induced sucrose preference deficits [39]. We have previously shown that deletion of the GluN2B receptor subtype from the BNST can mimic actions of ketamine [22]. Of note, recent work has shown that the antidepressant effect of ketamine may be due to its metabolite (2S, 6S;2R,6R)- HNK. Zanos and colleagues have shown that HNK is three-fold higher in female brain tissue after ketamine administration compared to male mice. The relative roles and actions of these metabolites remain an evolving literature currently [40-42].

Electrophysiological studies have shown that chronic noncontingent ethanol administration and withdrawal increases the levels and function of the GluN2B NMDAR subtype within the BNST [17]. In addition, we have shown that LTP, a form of plasticity, is dependent upon the presence of GluN2B within the BNST [23]. Here we show that animals with a history of chronic, continuous $2 \mathrm{BC}$ ethanol drinking and two-week abstinence have blunted BNST STP. This is reversed when ketamine is given at the beginning of abstinence, suggesting that ketamine may change the location and functionality of GluN2B subunit of the NMDARs. It is interesting to note that the STP inhibition noted here is very similar to the inhibition produced by acute alcohol administration to BNST slices [26]; however, it differs from the enhanced LTP seen of withdrawal from chronic intermittent alcohol vapor exposure [23]. In future studies it will be of interest to determine whether such differences relate to contingency, BEC levels, pyrazole supplementation, or other possibilities.

Withdrawal periods may be a critical factor in the development of affective disorders

Clinical studies have demonstrated that depression and alcohol use are highly comorbid. Different preclinical models of alcohol intake have shown that depression-like behavior develops from either 1 or 2 days after abstinence in a limited access drinking in the dark (DID) model [43], or in a more delayed fashion after abstinence from chronic continuous 2BC [3-5]. Animals that undergo a chronic DID paradigm are presented with limited access and the potential for multiple withdrawal periods since the ethanol is removed during the light phase. This pattern may hasten the development of affective disturbances. Lee et al. [43] found that male mice that underwent DID for 6 weeks and were tested in the EPM and light dark box at either day 1 or 2 of withdrawal or day 21 and 22 of withdrawal exhibited potential anxiety-like phenotypes. In future studies it will be of interest to directly compare these paradigms to determine the degree to which sex and ethanol availability regimen contribute to the differences observed.

To summarize, we report that forced abstinence from six-week long $2 \mathrm{BC}$ ethanol drinking induces anxiety and leads to the development of affective disturbances during abstinence. In addition, we show for the first time, to our knowledge, that ketamine can be used as an inoculant to prevent the development of ethanol dependent affective disturbances only if it is administered at a specific time point. The critical time point appears to be at the beginning of the abstinent period. This action may be due, in part, through ketamine enhancing plasticity within the BNST when administered at the onset of abstinence. The findings of this study should aid in uncovering the neurobiological bases of alcohol related affective disturbances, and aid in the development of more effective and longer lasting therapeutics $[44,45]$.

\section{ACKNOWLEDGEMENTS}

NIH grant support for this work was provided by R01 AA019455 (DGW), and T32 MH065215.

\section{ADDITIONAL INFORMATION}

Competing interests: The authors declare no competing interests.

Publisher's note: Springer Nature remains neutral with regard to jurisdictional claims in published maps and institutional affiliations.

\section{REFERENCES}

1. Lynskey MT. The comorbidity of alcohol dependence and affective disorders: treatment implications. Drug Alcohol Depend. 1998;52:201-9.

2. Heinz A, Ragan P, Jones DW, Hommer D, Williams W, Knable MB, et al. Reduced central serotonin transporters in alcoholism. Am J Psychiatry. 1998;155:1544-9.

3. Holleran KM, Wilson HH, Fetterly TL, Bluett RJ, Centanni SW, Gilfarb RA, et al. Ketamine and MAG lipase inhibitor-dependent reversal of evolving depressivelike behavior during forced abstinence from alcohol drinking. Neuropsychopharmacology. 2016;41:2062-71.

4. Pang TY, Du X, Catchlove WA, Renoir T, Lawrence AJ, Hannan AJ. Positive environmental modification of depressive phenotype and abnormal hypothalamic-pituitary-adrenal axis activity in female C57BL/6J mice during abstinence from chronic ethanol consumption. Front Pharmacol. 2013; 4:93 Published online 2013 july 23.

5. Pang TY, Renoir T, Du X, Lawrence AJ, Hannan AJ. Depression-related behaviours displayed by female $\mathrm{C} 57 \mathrm{BL} / 6 \mathrm{~J}$ mice during abstinence from chronic ethanol consumption are rescued by wheel-running. Eur J Neurosci. 2013b;37:1803-10.

6. Stevenson JR, Schroeder JP, Nixon K, Besheer J, Crews FT, Hodge CW. Abstinence following alcohol drinking produces depression-like behavior and reduced hippocampal neurogenesis in mice. Neuropsychopharmacology. 2009;34:1209-22.

7. Bardeleben $\mathrm{U}$, von, Heuser I, Holsboer F. Human $\mathrm{CRH}$ stimulation response during acute withdrawal and after medium-term abstention from alcohol abuse. Psychoneuroendocrinology. 1989;14:441-9.

8. Koob GF, Ahmed SH, Boutrel B, Chen SA, Kenny PJ, Markou A, et al. Neurobiological mechanisms in the transition from drug use to drug dependence. Neurosci Biobehav Rev. 2004;27:739-49.

9. Jaso BA, Niciu MJ, ladarola ND, Lally N, Richards EM, Park M, et al. Therapeutic modulation of glutamate receptors in major depressive disorder. Curr Neuropharmacol. 2016;1-14 https://doi.org/10.2174/1570159X14666160321123221.

10. Alén F, Serrano A, Gorriti MÁ, Pavón FJ, Orio L, Heras RGde, et al. The administration of atomoxetine during alcohol deprivation induces a time-limited increase in alcohol consumption after relapse. Int J Neuropsychopharmacol. 2014;17: 1905-10.

11. Gimeno C, Dorado ML, Roncero C, Szerman N, Vega P, Balanzá-Martínez V, et al. Treatment of comorbid alcohol dependence and anxiety disorder: review of the scientific evidence and recommendations for treatment. Front Psychiatry. 2017;8:173.

12. Silverstone $\mathrm{PH}$, Williams $\mathrm{R}$, McMahon L, Fleming R, Fogarty S. Alcohol significantly lowers the seizure threshold in mice when co-administered with bupropion hydrochloride. Ann Gen Psychiatry. 2008;7:11.

13. Fekadu A, Wooderson SC, Markopoulo K, Donaldson C, Papadopoulos A, Cleare AJ. What happens to patients with treatment-resistant depression? A systematic review of medium to long term outcome studies. J Affect Disord. 2009;116: 4-11.

14. Autry $A E$, Adachi $M$, Nosyreva E, Na ES, Los MF, Cheng PF, et al. NMDA receptor blockade at rest triggers rapid behavioural antidepressant responses. Nature. 2011:475:91-5.

15. Berton O, Nestler EJ. New approaches to antidepressant drug discovery: beyond monoamines. Nat Rev Neurosci. 2006;7:137-51.

16. Kiselycznyk C, Jury NJ, Halladay LR, Nakazawa K, Mishina M, Sprengel R, et al. NMDA receptor subunits and associated signaling molecules mediating antidepressant-related effects of NMDA-GluN2B antagonism. Behav Brain Res. 2015;287:89-95.

17. Kash TL, Baucum AJ, Conrad KL, Colbran RJ, Winder DG. Alcohol exposure alters NMDAR function in the bed nucleus of the Stria terminalis. Neuropsychopharmacology. 2009;34:2420-9.

18. Hardy PA, Chen W, Wilce PA. Chronic ethanol exposure and withdrawal influence NMDA receptor subunit and splice variant mRNA expression in the rat cerebral cortex. Brain Res. 1999;819:33-9.

19. Henniger MSH, Wotjak $C T$, Hölter SM. Long-term voluntary ethanol drinking increases expression of NMDA receptor $2 \mathrm{~B}$ subunits in rat frontal cortex. Eur J Pharmacol. 2003;470:33-6. 
20. Hu X-J, Follesa P, Ticku MK. Chronic ethanol treatment produces a selective upregulation of the NMDA receptor subunit gene expression in mammalian cultured cortical neurons. Mol Brain Res. 1996;36:211-8.

21. Follesa P, Ticku MK. Chronic ethanol treatment differentially regulates NMDA receptor subunit mRNA expression in rat brain. Mol Brain Res. 1995;29:99-106.

22. Louderback KM, Wills TS, Muglia LJ, Winder DG. Knockdown of BNST GluN2Bcontaining NMDA receptors mimics the actions of ketamine on novelty-induced hypophagia. Transl Psychiatry. 2013;3:e331.

23. Wills TA, Klug JR, Silberman Y, Baucum AJ, Weitlauf C, Colbran RJ, et al. GluN2B subunit deletion reveals key role in acute and chronic ethanol sensitivity of glutamate synapses in bed nucleus of the stria terminalis. Proc Natl Acad Sci USA. 2012;109:E278-87.

24. Pomrenze MB, Fetterly TL, Winder DG, Messing RO. The Corticotropin Releasing Factor Receptor 1 in Alcohol Use Disorder: Still a Valid Drug Target? Alcohol Clin Exp Res. 2017; https://doi.org/10.1111/acer.13507.

25. Vranjkovic O, Pina M, Kash TL, Winder DG. The bed nucleus of the stria terminalis in drug-associated behavior and affect: A circuit-based perspective. Neuropharmacology. 2017;122:100-6.

26. Weitlauf C, Egli RE, Grueter BA, Winder DG. High-frequency stimulation induces ethanol-sensitive long-term potentiation at glutamatergic synapses in the dorsolateral bed nucleus of the Stria terminalis. J Neurosci. 2004;24(25):5741-7.

27. Kash TL, Matthews RT, Winder DG. Alcohol inhibits NR2B-containing NMDA receptors in the ventral bed nucleus of the stria terminalis. Neuropsychopharmacology. 2008;33:1379-90.

28. Lee KM, Coehlo M, McGregor HA, Waltermire RS, Szumlinski KK. Binge alcohol drinking elicits persistent negative affect in mice. Behav Brain Res. 2015;291:385-98.

29. Bodnoff SR, Suranyi-Cadotte B, Aitken DH, Quirion R, Meaney MJ. The effects of chronic antidepressant treatment in an animal model of anxiety. Psychopharmacology. 1988;95:298-302.

30. Bodnoff SR, Suranyi-Cadotte B, Quirion R, Meaney MJ. A comparison of the effects of diazepam versus several typical and atypical anti-depressant drugs in an animal model of anxiety. Psychopharmacology. 1989;97:277-9.

31. Li N, Liu R-JJ, Dwyer JM, Banasr M, Lee B, Son H, et al. Glutamate N-methyl-Daspartate receptor antagonists rapidly reverse behavioral and synaptic deficits caused by chronic stress exposure. Biol Psychiatry. 2011;69:754-61.

32. McGowan JC, LaGamma CT, Lim SC, Tsitsiklis M, Neria Y, Brachman RA, et al. Prophylactic ketamine attenuates learned fear. Neuropsychopharmacology. 2017;42:1577-89.
33. Brachman RA, McGowan JC, Perusini JN, Lim SC, Pham TH, Faye C, et al. Ketamine as a prophylactic against stress-induced depressive-like behavior. Biol Psychiatry. 2016;79:776-86.

34. Raab-Graham KF, Workman ER, Namjoshi S, Niere F. Pushing the threshold: how NMDAR antagonists induce homeostasis through protein synthesis to remedy depression. Brain Res. 2016;1647:94-104.

35. Workman E, Niere F, Raab-Graham K. Engaging homeostatic plasticity to treat depression. Nat Publ Gr. 2017; doi.

36. Zanos P, Thompson SM, Duman RS, Zarate CA, Gould TD. Convergent mechanisms underlying rapid antidepressant action. CNS Drugs. 2018;32:197-227.

37. Li N, Lee B, Liu R-J, Banasr M, Dwyer JM, Iwata M, et al. mTOR-dependent synapse formation underlies the rapid antidepressant effects of NMDA antagonists. Science. 2010;329:959-64.

38. Maeng S, Zarate CA, Du J, Schloesser RJ, McCammon J, Chen G, et al. Cellular mechanisms underlying the antidepressant effects of ketamine: role of a-amino-3hydroxy-5-methylisoxazole-4-propionic acid receptors. Biol Psychiatry. 2008;63: 349-52.

39. Li S-X, Han Y, Xu L-Z, Yuan K, Zhang R-X, Sun C-Y, et al. Uncoupling DAPK1 from NMDA receptor GluN2B subunit exerts rapid antidepressant-like effects. Mol Psychiatry. 2018;23:597-608

40. Kavalali ET, Monteggia LM. The ketamine metabolite $2 \mathrm{R}, 6 \mathrm{R}-\mathrm{Hydroxynorketamine}$ blocks NMDA receptors and impacts downstream signaling linked to antidepressant effects. Neuropsychopharmacology. 2018;43:221-2.

41. Suzuki K, Nosyreva E, Hunt KW, Kavalali ET, Monteggia LM. Effects of a ketamine metabolite on synaptic NMDAR function. Nature. 2017;546:E1-3.

42. Zanos P, Moaddel R, Morris PJ, Georgiou P, Fischell J, Elmer Gl, et al. NMDAR inhibition-independent antidepressant actions of ketamine metabolites. Nature. 2016;533:481-6.

43. Lee KM, Coehlo MA, Solton NR, Szumlinski KK. Negative affect and excessive alcohol intake incubate during protracted withdrawal from binge-drinking in adolescent, but not adult, mice. Front Psychol. 2017;8:1128.

44. Liu R-J, Ota KT, Dutheil S, Duman RS, Aghajanian GK. Ketamine strengthens CRFactivated amygdala inputs to basal dendrites in mPFC layer $V$ pyramidal cells in the prelimbic but not infralimbic subregion, a key suppressor of stress responses. Neuropsychopharmacology. 2015;40 2066-75.

45. Zanos P, Moaddel R, Morris PJ, Georgiou P, Fischell J, Elmer Gl, et al. Zanos et al. reply. Nature. 2017;546:E4-5. 